\title{
High-density lipoprotein cholesterol is associated with multiple sclerosis fatigue: A fatigue-metabolism nexus?
}

\author{
Richard W. Browne, PhD*, Dejan Jakimovski, MD, Nicole Ziliotto, PhD, \\ Jens Kuhle, MD, PhD, Francesco Bernardi, MS, Bianca Weinstock-Guttman, MD, \\ Robert Zivadinov, MD, PhD, Murali Ramanathan, PhD
}

\begin{abstract}
Department of Biotechnical and Clinical Laboratory Sciences, University at Buffalo, State University of New York, Buffalo, NY, USA (Drs Browne and Ramanathan); Buffalo Neuroimaging Analysis Center, Department of Neurology, Jacobs School of Medicine and Biomedical Sciences, University at Buffalo, State University of New York, Buffalo, NY, USA (Drs Jakimovski and Zivadinov); Department of Life Sciences and Biotechnology, University of Ferrara, Ferrara, Italy (Drs Ziliotto and Bernardi); Neurologic Clinic and Policlinic, Departments of Medicine, Biomedicine and Clinical Research, University Hospital Basel, University of Basel, Basel, Switzerland (Dr Kuhle); Department of Neurology, University at Buffalo, State University of New York, Buffalo, NY, USA (Drs Weinstock-Guttman and Zivadinov); and Department of Pharmaceutical Sciences, University at Buffalo, State University of New York, Buffalo, NY, USA (Dr Ramanathan)
\end{abstract}

KEYWORDS:

Multiple sclerosis;

Fatigue;

Cholesterol;

HDL;

Neurofilaments
BACKGROUND: Fatigue is a frequent symptom in multiple sclerosis (MS). The role of cholesterol and lipids in MS fatigue has not been investigated.

OBJECTIVE: To investigate the associations of cholesterol biomarkers and serum neurofilament light chain (sNfL) with fatigue in relapsing-remitting MS.

METHODS: This cross-sectional study included 75 relapsing-remitting MS patients $(69 \%$ female, mean age \pm SD: $49.6 \pm 11$ years and median Expanded Disability Status Scale score: 2.0). Fatigue, disability, and depression were assessed with Fatigue Severity Scale (FSS), Expanded Disability Status Scale, and the Beck Depression Index-Fast Screen, respectively. sNfL was measured using singlemolecule array technology. Plasma total cholesterol (TC), high-density lipoprotein cholesterol (HDL-C), and an apolipoprotein panel data were obtained. Soluble intercellular adhesion molecule1 (sICAM-1), soluble vascular adhesion molecule-1 (sVCAM-1), chemokine (C-C motif) ligand 5 (CCL5 or RANTES), and CCL18 levels were measured to assess inflammation.

RESULTS: The mean FSS was $4.27 \pm 1.73$, and $57 \%$ had severe fatigue status (SFS, FSS $\geq 4.0$ ). In regression analyses adjusted for age, sex, disability, and depression, lower FSS and SFS were associated with greater HDL-C ( $P=.006$ for FSS, and $P=.016$ for SFS) and lower TC to HDL-C ratio $(P=.011$ for FSS, and $P=.009$ for SFS). Apolipoprotein A-II was also associated with FSS $(P=.022)$. sNfL, CCL5, CCL18, sICAM-1, and sVCAM-1 levels were not associated with fatigue
* Corresponding author. Department of Biotechnical and Clinical Laboratory Sciences, State University of New York, 311 Farber Hall, Buffalo, NY 14214-8033, USA.
E-mail address: rwbrowne@buffalo.edu

Submitted March 12, 2019. Accepted for publication June 4, 2019. 
after adjusting for disability and depression.

CONCLUSIONS: TC to HDL-C ratio is associated with MS fatigue. Our results implicate a potential role for the HDL-C pathway in MS fatigue and could provide possible targets for the treatment of MS fatigue.

(C) 2019 National Lipid Association. All rights reserved.

\section{Introduction}

Multiple sclerosis (MS) is an inflammatory, demyelinating disease that causes lesion formation and atrophy. MS results in physical and cognitive disability. Fatigue is a very frequent and debilitating symptom for patients with MS. ${ }^{1-3}$ It affects $>75 \%$ of patients with MS, and it is associated with diminished quality of life independently of disability. ${ }^{4}$

Operationally, fatigue is defined as "a subjective lack of physical and/or mental energy that is perceived by the individual or caregiver to interfere with usual or desired activities." 5 Patients with MS experience acute fatigue, which results in "activity-limiting sluggishness" and also "lassitude, a chronic, persistent extreme feeling of tiredness" unrelated to overall activity level. ${ }^{6}$

MS fatigue is multifactorial and results from a combination of pathobiological and psychological factors ${ }^{7,8}$ and is associated with disability, ${ }^{9}$ but not age or disease duration. ${ }^{9,10}$ Fatigue can be a consequence of depression, ${ }^{8,11}$ which is frequent in patients with MS. However, fatigue also occurs in patients with MS who are not depressed ${ }^{7}$ and in patients with benign $\mathrm{MS}^{12}$ and may independently influence disease progression. ${ }^{13,14}$ The pathophysiology of fatigue in neurological disorders such as MS can involve contributions from injury to the central, peripheral, and autonomic nervous systems, to the neuromuscular junction or muscle metabolism and function. ${ }^{15}$

Pharmacological options for treating MS-associated fatigue are limited. The available treatment strategies are symptomatic or rehabilitative. ${ }^{15}$ MS patients with fatigue are commonly prescribed modafinil ${ }^{16}$ or amantadine. ${ }^{17}$ Pharmaceutical antifatigue agents have mostly a nonspecific stimulant activity and are often associated with side effects. In addition, there is also support for the usefulness of exercise and educational interventions such as physical therapy with vestibular rehabilitation and cognitive behavioral therapy in treating MS fatigue. ${ }^{18}$

Serum neurofilament light chain (sNfL) levels are a novel and highly promising state-of-the-art biomarker for active neurological disease and neurodegeneration that can now be measured in blood using highly sensitive singlemolecule array technology. ${ }^{19}$ Neurofilaments are cytoskeletal proteins present in neurons that are released into cerebrospinal fluid when neuronal cell damage occurs and diffuse into blood. sNfL has been shown to be a highly specific biomarker for neuronal cell damage in MS in several clinical studies. sNfL levels can distinguish patients with MS from healthy controls and MS patients with contrastenhancing lesions from those without contrast-enhancing lesions, and it is a predictor of brain atrophy and disease worsening on the Expanded Disability Status Scale (EDSS). ${ }^{20}$ However, it has not been investigated extensively in the context of MS fatigue.

We investigated the associations of lipid biomarkers based on the working hypothesis that lipids might modulate MS fatigue via effects on the MS disease process as well as through effects on muscle metabolism and inflammationinduced interactions between neurovascular cells and the immune system. An emerging body of evidence has demonstrated that metabolic changes ${ }^{21,22}$ underlie the immune and neurodegenerative pathophysiological processes of MS and cholesterol biomarkers are associated with measures of brain injury and disease progression in both established MS and following the first demyelinating event. ${ }^{23-25}$

A more extensive understanding of the associations, if any, of lipid pathways with MS fatigue could facilitate the identification of targets for drug treatments and better management strategies. Therefore, the purpose of this research was to systematically assess the associations of MS fatigue with blood biomarkers of nervous system injury and lipid metabolism.

\section{Methods}

\section{Study population}

The study data were obtained from a prospective study of clinical, genetic, and environmental risk factors in MS at the MS Center of the State University of New York at Buffalo. ${ }^{26}$ Written informed consent was collected from all participants, and Human Subjects Institutional Review Board of the University at Buffalo approved the study protocol.

The enrolled patients underwent neurological examinations. Fatigue was assessed using the Fatigue Severity Scale (FSS). ${ }^{27}$ Disability was assessed using the EDSS. ${ }^{28}$ Depression was assessed using the Beck Depression Index-Fast Screen (BDI-FS) scale values. ${ }^{29,30}$ Body mass index (BMI) was computed as weight (in $\mathrm{kg}$ ) divided by height (in meters) squared. Blood samples were collected for analyses of biomarkers.

This substudy included 75 relapsing-remitting MS patients with available FSS scores, neurofilament measurements, and serum cholesterol biomarkers.

Plasma samples for lipid, apolipoprotein, sNfL, cytokine, and inflammatory biomarker analyses were drawn from nonfasted patients into Vacutainer tubes containing EDTA anticoagulant (Becton-Dickinson, Franklin Lakes, NJ). Plasma samples were stored at $-80^{\circ} \mathrm{C}$ until use. The 
median time from blood draw date to sNfL analysis was 2.7 years. All analysts were blinded to patients' clinical status.

\section{Blood lipid and apolipoprotein profile}

Plasma total cholesterol (TC) and high-density lipoprotein cholesterol (HDL-C) were measured using FDAapproved diagnostic reagent kits and quality control materials (Sekisui Diagnostics) on an ABX Pentra 400 automated chemistry analyzer. Low-density lipoprotein cholesterol (LDL-C) was obtained with the Friedewald equation. ${ }^{31}$ Assay coefficients of variation $(\mathrm{CVs})$ are $<5 \%$.

Apolipoproteins (ApoA-I, ApoA-II, ApoB, ApoC-II, and ApoE) were analyzed by immunoturbidimetric methodology using FDA-approved diagnostic kits and quality control materials (Kamiya Biomedical). All assays were done on the ABX Pentra 400 automated chemistry analyzer. The assay CVs are $<5 \%$.

\section{Serum neurofilaments}

Serum neurofilament light chain levels were obtained using the highly sensitive state-of-the-art single molecule array assay as previously described (analytical sensitivity $0.32 \mathrm{pg} / \mathrm{mL}){ }^{32}$ The between-run and within-run CVs are $<8 \%$. The sNfL analyses were conducted at the University Hospital, Basel, Switzerland.

\section{Cytokines and inflammatory biomarkers}

Soluble intercellular adhesion molecule-1 (sICAM-1), soluble vascular adhesion molecule-1 (sVCAM-1) levels, and chemokine (C-C motif) ligand 5 (CCL5, also known as RANTES for activation, normal $\mathrm{T}$ cell expressed and secreted, or RANTES) were assayed using Milliplex magnetic bead kits (human neurodegenerative disease panel 3, HNDG3MAG-36K; Merck Millipore, Germany). Concentrations $(\mathrm{ng} / \mathrm{mL})$ were calculated from a target-specific standard curve. The calculated interassay CVs for sICAM1, sVCAM-1, and CCL5 were $5.7 \%, 7.3 \%$, and $4.7 \%$, respectively. CCL18 levels were measured using Luminex Screening Assays magnetic bead kits (Luminex R\&D Systems Inc, Minneapolis, MN). Data were acquired using the Luminex 100 system and analyzed using the Bio-Plex Manager software, version 6.0 (both from Bio-Rad Laboratories, Hercules, CA).

\section{Data analysis}

Statistical analyses were conducted with the SPSS statistical program (version 24). sNfL, CCL5, and CCL18 values were logarithm (base 10) transformed to reduce skew. A severe fatigue status (SFS) binary variable was created based on FSS $\geq 4.0$. This threshold was based on a psychometric study of FSS. ${ }^{33}$ A depression status variable was defined based on BDI-FS $\geq 4.0 .^{29,34}$ The overweight or obese status variable was defined based on BMI $\geq$ $25 \mathrm{~kg} / \mathrm{m}^{2}$, and the TC to HDL-C $\geq 3.5$ status variable was defined based on the thresholds established in cardiovascular disease. ${ }^{35,36}$ The binary interferon-beta treatment status and statin treatment status variables were assigned a value of 1 if the patient was receiving treatment with any interferon-beta product or statin, respectively, and a value 0 otherwise.

FSS and SFS were analyzed as the dependent variables in linear regression and logistic regression, respectively. All the linear and logistic regression analyses included age, sex, EDSS and BDI-FS scores, and the individual blood biomarker of interest (log-transformed sNfL level, TC, HDL-C, LDL-C, ApoA-I, ApoA-II, ApoB, ApoC-II, or ApoE) as predictors. BMI was also assessed using the same regression framework. Subsequently, stepwise regression with all these variables as input predictors was used to identify the most parsimonious combination of variables capable of explaining FSS and SFS. The $P$-values for variable entry and variable removal were .05 and .10 , respectively.

To better delineate the role of sNfL in MS fatigue, we conducted an additional set of linear and logistic regression analyses of the FSS and SFS dependent variables with age, sex, and log-transformed sNfL level as predictors. In these analyses, EDSS and BDI-FS were excluded and then added individually.

BMI, interferon-beta treatment, ${ }^{37,38}$ and statin treatment can also affect cholesterol profiles. In secondary analyses, FSS and SFS were analyzed as dependent variables in linear regression and logistic regression analyses, respectively, with age, sex, EDSS, BDI-FS score, BMI, interferon-beta and statin treatment status, and the individual blood biomarker of interest (log-transformed sNfL level, TC, HDL-C, LDL-C, ApoA-I, ApoA-II, ApoB, ApoC-II, or ApoE) as predictors. Stepwise regression analyses as previously described were also conducted.

The associations of the cytokines and inflammatory biomarkers with FSS and SFS as the dependent variables were analyzed in linear regression and logistic regression, respectively. All the linear and logistic regression analyses included age, sex, EDSS and BDI-FS scores, and the individual cytokine or inflammatory biomarker of interest (log-transformed CCL5, log-transformed CCL18, ICAM-1, or VCAM-1) as predictors.

We conducted receiver operating characteristic (ROC) curve analyses of the log-transformed sNfL level, cholesterol to HDL-C ratio, EDSS, and BDI-S variables to identify the optimal thresholds at which they could be used to predict SFS. The area under the curve (AUC) of the ROC curve and its asymptotic significance under the null hypothesis of $\mathrm{AUC}=0.5$ were assessed. The point on the ROC curve with maximum vertical distance from the diagonal is typically considered the optimal threshold if sensitivity and specificity are equally important. 


\section{Results}

\section{Demographic and clinical characteristics}

The demographic and clinical characteristics of the 75 relapsing-remitting MS patients are summarized in Table 1.

The majority of the patients with MS (57\%) had severe fatigue as indicated by FSS $\geq 4.0$. The proportion of patients with EDSS $\geq 4.0$ was $15 \%$. The proportion of patients with MS who were overweight or obese was $70 \%$ and $33 \%$, respectively, had BDI-FS $\geq 4.0$, which is suggestive of depression. The percentage of statin-treated patients was $15 \%(11 / 75)$.

Table 1 Demographic and clinical characteristics of the RRMS patients

\begin{tabular}{|c|c|}
\hline Demographics & RR-MS \\
\hline Females:Males ( $\%$ Female) & $52: 23(69 \%)$ \\
\hline Relapsing-remitting disease course & $75(100 \%)$ \\
\hline \multicolumn{2}{|l|}{ Race/ethnicity (\%) } \\
\hline Caucasian & $71(95 \%)$ \\
\hline African-American & $2(3 \%)$ \\
\hline Hispanic/Latino & $1(1 \%)$ \\
\hline Asian & $1(1 \%)$ \\
\hline Other & - \\
\hline Age, y & $49.6 \pm 11$ \\
\hline Disease duration, y & $17.0 \pm 9.1$ \\
\hline \multicolumn{2}{|l|}{ Disease-modifying treatments } \\
\hline No disease-modifying treatment & $12 \%$ \\
\hline Interferons $^{\S}$ & $32 \%$ \\
\hline Glatiramer acetate & $28 \%$ \\
\hline Natalizumab & $7 \%$ \\
\hline Other oral disease-modifying treatments ${ }^{\ddagger}$ & $15 \%$ \\
\hline Other drugs & $5 \%$ \\
\hline Missing information & $1 \%$ \\
\hline FSS score & $4.27 \pm 1.73$ \\
\hline FSS Score $\geq 4.0$ & $43 / 75(57 \%)$ \\
\hline Median EDSS (interquartile range)* & $2.0(1.6)$ \\
\hline$E D S S \geq 4.0$ & $11 / 74(15 \%)$ \\
\hline BDI-FS & $2.6 \pm 3.0$ \\
\hline $\mathrm{BDI}-\mathrm{FS} \geq 4.0$ & $25 / 75(33 \%)$ \\
\hline BMI, $\left.\mathrm{kg} / \mathrm{m}^{2}\right)^{*}$ & $28.2 \pm 6.2$ \\
\hline $\mathrm{BMI} \geq 25 \mathrm{~kg} / \mathrm{m}^{2}$ & $59 / 73(70 \%)$ \\
\hline
\end{tabular}

BDI-FS, Beck Depression Index-Fast Screen; BMI, body mass index; EDSS, Expanded Disability Status Scale; FSS, Fatigue Severity Scale.

All continuous variables are shown as mean \pm SD. The ordinal EDSS is shown as median (interquartile range). Categorical variables are shown as count/total count (\%).

*EDSS was missing for 1 patient, and BMI was missing for 2 patients.

$\S$ Interferons include intramuscular, subcutaneous interferon-beta$1 \mathrm{a}$ and peginterferon-beta-1a.

$\ddagger 0$ ther oral disease-modifying drugs include fingolimod, dimethyl fumarate, and teriflunomide. Other drug categories include intravenous immunoglobulin, methotrexate, and mitoxantrone.

\section{Biomarker results}

The sNfL, lipid profile (TC, HDL-C, LDL-C, TC to HDL-C ratio), and apolipoprotein profile (ApoA-I, ApoAII, ApoB, ApoC-II, and ApoE) results are summarized in Table 2.

FSS was associated with EDSS (partial correlation $\left.r_{p}=.28, P=.017\right)$ and BDI-FS $\left(r_{p}=.26, P=.030\right)$ in linear regression analyses that were adjusted for age $\left(r_{p}=-0.027, P=.83\right)$ and $\operatorname{sex}\left(r_{p}=-0.18, P=.14\right)$. These results are expected from prior research. However, SFS was not associated with $\operatorname{EDSS}[\operatorname{Exp}(B)=1.46$, $P=.099]$ or BDI-FS $[\operatorname{Exp}(B)=1.11, P=.24]$ in logistic regression analyses that were adjusted for age $[\operatorname{Exp}(B)=0.98, P=.32]$ and $\operatorname{sex}[\operatorname{Exp}(B)=2.22, P=.15]$.

Table 3 summarizes the regression results for the FSS and SFS with sNfL, lipid profile and apolipoprotein profile biomarkers in analyses that were adjusted for age, sex, EDSS, and BDI-FS. Greater HDL-C values $(P=.006$ for FSS and $P=.016$ for SFS) and lower TC to HDL-C ratio values ( $P=.011$ for FSS and $P=.009$ for SFS) were associated with lower FSS and SFS. ApoA-II, an apolipoprotein associated with HDL-C, was associated with FSS $(P=.022)$. The other lipid and apolipoprotein biomarkers were not associated with FSS or SFS. sNfL was not associated with FSS or SFS.

Because disability and MS-related depression can be caused by the MS disease process, we evaluated whether these variables were masking the associations of sNfL with fatigue in the regression analyses. We conducted additional regression analyses of FSS and SFS with sex and age and sNfL as predictors, ie, with EDSS and BDI-FS not included. These regression analyses exposed SNfL associations with FSS $\left(r_{p}=.19, P=.007\right)$ and a nonsignificant trend with SFS $[\operatorname{Exp}(B)=2.73, P=.055]$. However, when either EDSS or BDI-FS were present in the predictor set, sNFL was not associated with FSS or SFS (data not shown).

To identify the "best" subset of variables capable of modeling FSS and SFS, we conducted stepwise linear regression that included age, sex, EDSS, BDI-FS, and all the biomarkers in Table 3. The final linear regression model for FSS contained EDSS $\left(r_{p}=.28, P=.018\right)$, BDI-FS $\left(r_{p}=.26, P=.031\right)$, and TC to HDL-C ratio $\left(r_{p}=.29\right.$, $P=.015)$. The final logistic regression model for SFS contained $\operatorname{EDSS}[\operatorname{Exp}(B)=1.47, P=.058]$ and TC to HDL-C ratio $[\operatorname{Exp}(B)=2.57, P=.016]$.

Because BMI, interferon-beta treatment status, and statin treatment status can affect lipid profiles, we conducted additional regression analyses with these predictors also included. The results are summarized in Supplementary Table 1. The associations of HDL-C and TC to HDL-C ratio were significant. The final linear regression model for FSS contained EDSS $\left(r_{p}=.29, P=.016\right)$, BDI-FS $\left(r_{p}=.28, P=.022\right)$, TC to HDL-C ratio $\left(r_{p}=.34\right.$, $P=.006)$, and interferon-beta treatment status $\left(r_{p}=-0.25, P=.046\right)$. The final logistic regression model 


\section{Table 2 Summary of biomarkers}

\begin{tabular}{|c|c|}
\hline Biomarkers & Mean $\pm S D$ \\
\hline Neurofilament levels (sNfL), pg/mL & $18.3(12.6)^{*}$ \\
\hline $\log _{10}$ transformed sNfL levels & $1.24 \pm 0.30$ \\
\hline Total cholesterol (TC), mg/dL & $228 \pm 36$ \\
\hline Triglycerides, mg/dL* & $159 \pm 91$ \\
\hline $\begin{array}{l}\text { High-density lipoprotein } \\
\text { cholesterol (HDL-C), mg/dL }\end{array}$ & $59.8 \pm 14$ \\
\hline $\begin{array}{l}\text { Low-density lipoprotein } \\
\text { cholesterol (LDL-C), mg/dL* }\end{array}$ & $136 \pm 34$ \\
\hline Total cholesterol to HDL-C ratio & $3.91 \pm 0.76$ \\
\hline Apolipoprotein A-I (ApoA-I), mg/dL & $166 \pm 28$ \\
\hline Apolipoprotein A-II (ApoA-II), mg/dL & $43.5 \pm 7.0$ \\
\hline Apolipoprotein B (ApoB), mg/dL & $101 \pm 27$ \\
\hline Apolipoprotein C-II (ApoC-II), mg/dL & $4.82 \pm 1.8$ \\
\hline Apolipoprotein E (ApoE), mg/dL & $5.84 \pm 1.5$ \\
\hline \multicolumn{2}{|c|}{$\begin{array}{l}\text { All values shown are mean } \pm \text { SD except for } \mathrm{sNfL} \text {, which are median } \\
\text { (interquartile range). } \\
\text { *Low-density lipoprotein was calculated using the Friedewald } \\
\text { equation. One subject had triglycerides }>400 \mathrm{mg} / \mathrm{dL} \text {. No additional } \\
\text { adjustment was made to the low-density lipoprotein cholesterol calcu- } \\
\text { lation for this individual. }\end{array}$} \\
\hline
\end{tabular}

for SFS contained $\operatorname{EDSS}[\operatorname{Exp}(B)=1.54, P=.04]$ and TC to $\mathrm{HDL}-\mathrm{C}$ ratio $[\operatorname{Exp}(B)=2.77, P=.011]$.

To assess the contributions, if any, of inflammatory processes, we also examined the associations of FSS and SFS with two cytokines from the chemokine family, CCL5 or RANTES and CCL18, and two adhesion molecules, sICAM-1 and sVCAM-1. We did not find evidence for associations of FSS and SFS with any of these biomarkers (Supplementary Table 2).

Figure 1 shows the dependence of the proportion of patients with severe fatigue on TC to HDL-C ratio $\geq 3.5$ status. The proportion of patients with severe fatigue was twice as high in the TC to HDL-C ratio $\geq 3.5$ group (72\%) compared with the TC to HDL-C ratio $<3.5$ group (36\%).

The AUC of the ROC curve for TC to HDL-C ratio was $0.67 \pm \mathrm{SE} 0.066(P=.014)$, whereas the AUC $(0.55 \pm \mathrm{SE}$ $0.069)$ for $\log$-transformed sNFL was not $(P=.48)$. The ROC curves for log-transformed SNFL and TC to HDL-C ratio are summarized in Figure 2. Based on the sum of sensitivity and specificity (Fig. 2B), optimal threshold for TC to HDL-C ratio for SFS status was 3.53, which is close to the threshold used in cardiovascular disease. ${ }^{35,36}$ The sensitivity and specificity at this value were 0.79 and 0.41 , respectively. The AUC of the ROC curve for EDSS was $0.66 \pm \operatorname{SE} 0.065(P=.020)$, whereas the AUC $(0.61 \pm$ SE 0.066$)$ for BDI-FS was not significant $(P=.12)$.

\section{Discussion}

In this study of MS fatigue, we assessed whether sNfL and several cholesterol pathway biomarkers were associated with MS fatigue. We considered sNfL because it is a useful biomarker of neurodegeneration and nervous system injury, whereas the cholesterol pathway biomarkers provide clinically useful measures of metabolic dysregulation. MS fatigue as measured by the FSS and SFS was associated with specific cholesterol pathway biomarkers related to HDL-C in regression analyses that were adjusted for disability (as measured by the EDSS) and depression (as measured by the BDI-FS). The TC to HDL-C ratio was the most useful biomarker among the several cholesterol pathway biomarkers investigated. sNfL was not associated with fatigue on adjusting for disability and depression, but associations with FSS were exposed when disability and depression were not included.

Table 3 Regression results for the FSS and SFS with sNfL, lipid profile, apolipoprotein profile, cytokine, and inflammatory biomarkers

\begin{tabular}{lcc}
\hline & $\begin{array}{c}\text { FSS } \\
\text { Biomarker }\end{array}$ & $\begin{array}{c}\text { Linear regression } \\
r_{p}(P \text {-value })\end{array}$ \\
\hline Log $_{10}$ transformed sNfL levels & $0.029(.81)$ & $0.75(.78)$ \\
Body mass index (BMI), kg/m ${ }^{2}$ & $0.11(.39)$ & $1.06(0.21)$ \\
Total cholesterol (TC), mg/dL & $-0.10(.40)$ & $0.996(.58)$ \\
High-density lipoprotein cholesterol (HDL-C), mg/dL & $0.95(.016)$ \\
Low-density lipoprotein cholesterol (LDL-C), mg/dL & $-0.32(.006)$ & $0.98(.52)$ \\
Apolipoprotein A-I (ApoA-I), mg/dL & $-0.088(.48)$ & $0.98(.07)$ \\
Apolipoprotein A-II (ApoA-II), mg/dL & $-0.23(.056)$ & $0.93(.08)$ \\
Apolipoprotein B (ApoB), mg/dL & $-0.27(.022)$ & $1.01(.58)$ \\
Apolipoprotein C-II (ApoC-II), mg/dL & $0.056(.65)$ & $1.19(.24)$ \\
Apolipoprotein E (ApoE), mg/dL & $0.11(.36)$ & $1.15(.42)$ \\
Total cholesterol to HDL-C ratio & $0.096(.43)$ & $2.91(.009)$ \\
\hline
\end{tabular}

BDI-FS, Beck Depression Index-Fast Screen; BMI, body mass index; EDSS, Expanded Disability Status Scale; FSS, Fatigue Severity Scale. All regression analyses adjusted for age, sex, and EDSS and BDI-FS scores.

The partial correlation $\left(r_{p}\right)$ values and the corresponding $P$-values are shown for each biomarker for FSS, whereas $\operatorname{Exp}(B)$ and $P$-values are shown for the SFS. 


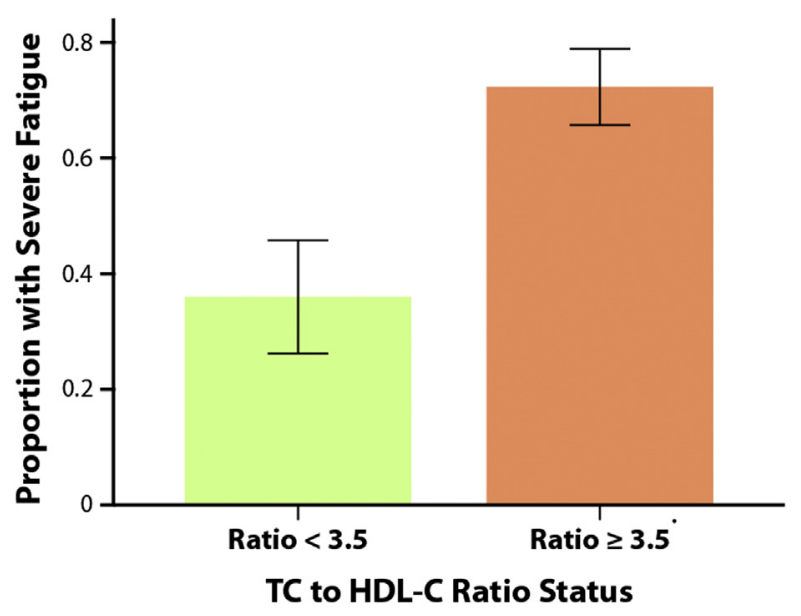

Figure 1 The proportion of relapsing-remitting multiple sclerosis patients with severe fatigue as defined by Fatigue Severity Scale score $\geq 4.0$ on total cholesterol (TC) to high-density lipoprotein cholesterol (HDL-C) ratio $\geq 3.5$ status. The bars represent the proportion, and the error bars represent the standard error of the proportion.

We found the expected associations with disability and depression, but our results additionally identified key associations between TC to HDL-C ratio and MS fatigue, which we attribute primarily to the HDL-C pathway. The TC to HDL-C ratio is considered to be a more effective predictor of cardiovascular disease risk than other lipid profile biomarkers such as TC or LDL-C alone. ${ }^{36}$

Based on the underlying causes, MS fatigue is sometimes categorized as central or primary fatigue and as peripheral or secondary fatigue. ${ }^{39}$ Primary fatigue is viewed as a result of the MS pathobiological process, which causes blood-brain barrier breakdown, central nervous system inflammation, demyelination, lesion formation, and neurodegeneration. The fatigue associated with heat sensitivity in patients with MS is an example of primary fatigue. Secondary fatigue is often viewed as the result of comorbidities such as depression, physical and emotional stress, and external factors, eg, lack of sleep. In clinical practice, it is not possible to distinguish between or measure the primary and secondary components of fatigue independently in patients with MS.

Figure 3 is a schematic of the proposed pathophysiological mechanisms via which the cholesterol pathway could potentially modulate MS fatigue. The schematic highlights the known effects of LDL-C and HDL-C on the inflammatory, vascular, and neurodegenerative aspects of the MS disease process as well as its effects on muscle. We hypothesize that skeletal muscle could be an important but overlooked organ for potential interactions between metabolism and fatigue because it is a key effector organ at which MS physical disability, fatigue, and weakness manifest to patients. Muscle cells, which have high mitochondrial density to support the energy demands of mechanical force production for physical activity, are important endogenous sources of reactive oxygen species. ${ }^{46}$ Reactive oxygen species oxidatively modify cholesterol,
A

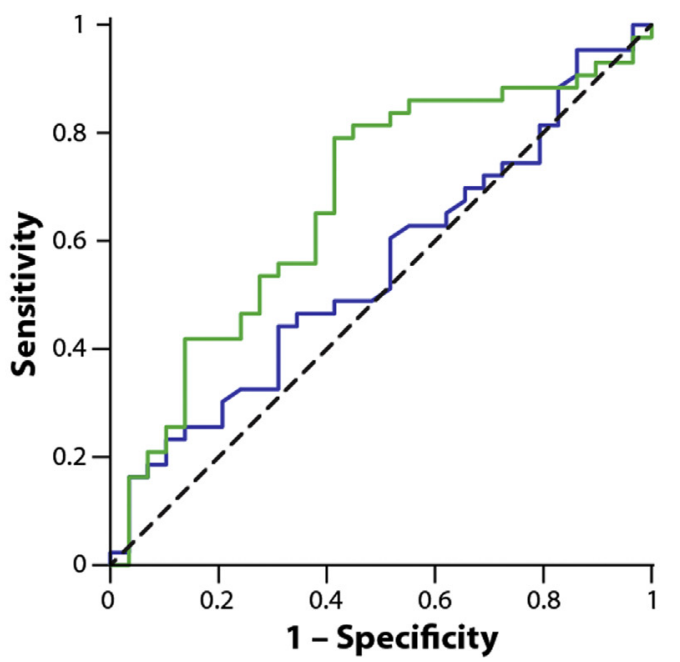

B

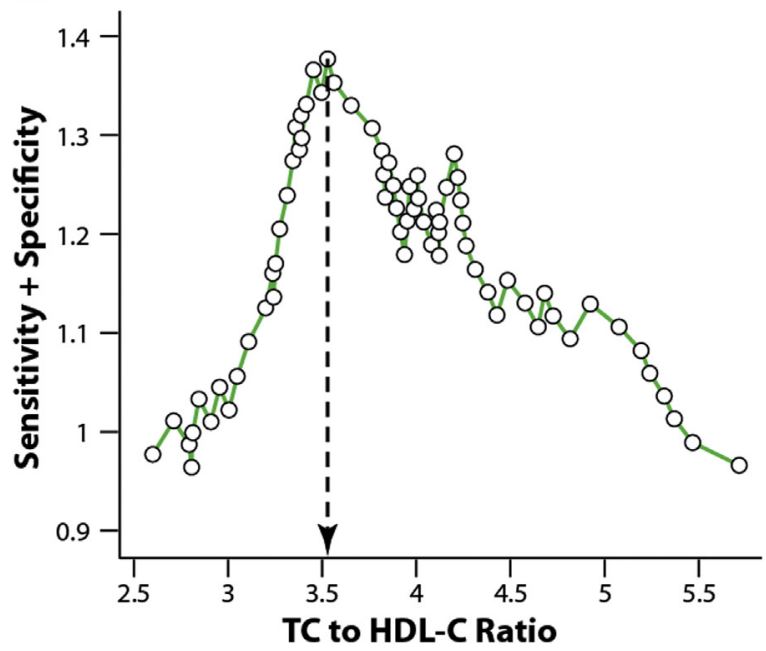

Figure 2 (A) The ROC curves for the SFS variable with TC to HDL-C ratio (green line) and log-transformed neurofilament levels (blue line). The dashed line is the reference diagonal. (B) Sensitivity + specificity values from the ROC curve analysis of SFS for different values of TC to HDL-C ratio. The dashed arrow represents the TC to HDL-C ratio value for which the sensitivity + specificity is maximal. HDL-C, high-density lipoprotein cholesterol; ROC, receiver operating characteristic; SFS, severe fatigue status; TC, total cholesterol

unsaturated fatty acids, and LDL to produce proinflammatory mediators such as oxysterols, oxylipins, and oxidizedLDL. HDL-C exerts antiinflammatory effects, and it possesses antioxidant activities that rapidly hydrolyze lipid peroxides and protects lipoproteins from oxidative modifications. ${ }^{47,48}$ Furthermore, the critical role of cholesterol in muscle function is emerging in recent research. ${ }^{49,50}$ HDL-C and its signature apolipoprotein, ApoA-I, stimulate glucose uptake ${ }^{51}$ and increase respiration in muscle mitochondria. ${ }^{52}$ Muscle membrane cholesterol modulates the insertion of the glucose transporter GLUT-4, which mediates insulin-dependent glucose uptake into muscle. ${ }^{49}$ Among individuals 80 years of age and older, HDL-C is a reliable marker of frailty, muscle strength, and functional 


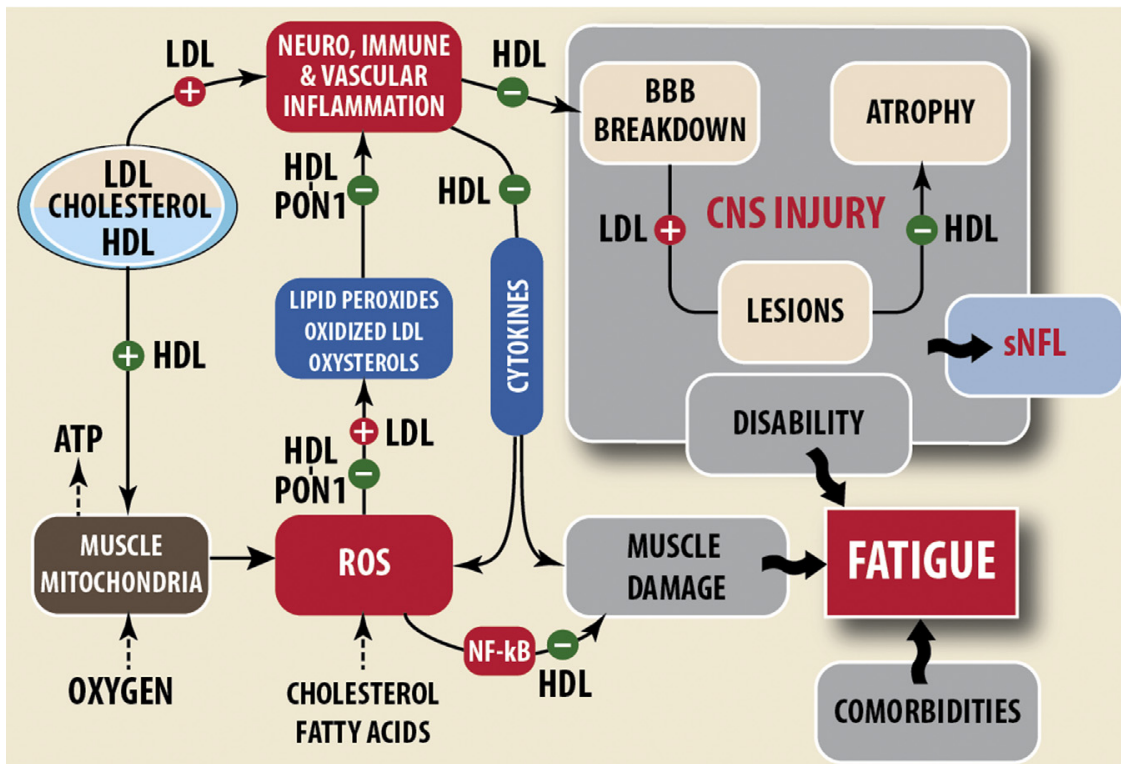

Figure 3 A schematic of the mechanisms via which the cholesterol pathway could modulate MS fatigue. The known effects of LDL-C and HDL-C on the inflammatory, vascular, and neurodegenerative aspects of the MS disease process and effects on muscle are highlighted. Muscle cells have high mitochondrial density to produce ATP to meet physiological energy demands of mechanical force production for physical activity and are an important endogenous source of ROS. ROS can oxidatively modify fatty acids, cholesterol, and LDL-C to produce inflammatory mediators such as lipid peroxides, oxysterols, and oxidized-LDL. ${ }^{40}$ ROS induce the NF-kB and other pathways, ${ }^{41-43}$ which can directly promote protein catabolism that lead to muscle weakness and atrophy. ${ }^{44,45}$ HDL-C has antiinflammatory effects and its antioxidant enzyme PON1 rapidly hydrolyzes lipid peroxides and protects lipoproteins from oxidative modifications. Higher HDL-C levels are associated with lower levels of BBB breakdown and gray matter atrophy. ${ }^{25,37}$ Higher LDL-C levels are associated with the formation of new T2 lesions. ${ }^{24,25}$ Serum neurofilaments (sNfL) are produced in response to active disease and neurodegeneration in MS. The plus and minus signs indicate activating and inhibitory associations, respectively. ATP, adenosine triphosphate; BBB, blood-brain barrier; HDL-C, high-density lipoprotein cholesterol; LDL-C, low-density lipoprotein cholesterol; MS, multiple sclerosis; NF-kB, nuclear factorkappa B; PON1, paraoxonase-1; ROS, reactive oxygen species; sNFL, serum neurofilament light chain.

status. ${ }^{53,54}$ We suggest that further study of muscle in MS fatigue is warranted.

Serum neurofilament light chain is rapidly emerging as a promising biomarker for nervous system injury in MS and other neurological diseases. ${ }^{55}$ Figure 3 shows that $\mathrm{SNfL}$ is associated with lesional injury and brain atrophy in MS. We intended SNfL to be a biomarker of nervous system tissue damage by MS disease processes, ${ }^{55}$ but the sNfL associations with FSS were exposed only when disability and depression were removed from the models. In contrast, the TC to HDL-C ratio consistently emerged as a salient fatigue-associated variable even when EDSS and BDI-FS were included.

Currently, magnetic resonance imaging (MRI) provides some of the best clinically useful biomarkers of MS pathology and disease progression. However, it has been challenging to identify conventional MRI biomarkers for fatigue. Fatigue and fatigue changes were not associated with contrast-enhancing lesion activity. ${ }^{56}$ Biberacher et al. ${ }^{57}$ found that total and cortical gray matter were associated with fatigue, but the associations were not significant on adjusting for disability. However, Marrie et al. ${ }^{58}$ found worsening fatigue associated with longitudinal changes in brain atrophy as measured by the brain parenchymal fraction. Using functional MRI, Filippi et al. ${ }^{59}$ demonstrated altered patterns of activation in the cortical and subcortical regions of the brain in fatigued MS patients. Fuchs et al. ${ }^{60}$ reported that disruption of white matter tracts between brain regions was negatively associated with fatigue.

It has been suggested that fatigue results from the ongoing inflammatory milieu, which results in higher levels of proinflammatory cytokines in MS. Heesen et al. found associations between whole blood-stimulated production of the inflammatory cytokines, tumor necrosis factor-alpha and interferon-gamma, and fatigue; cortisol and adrenocorticotropic hormone were not associated. However, Giavannoni et al. ${ }^{12}$ did not find any associations with several inflammatory markers including urinary neopterin, serum C-reactive protein, and soluble intercellular adhesion molecule-1 levels. Recently, Hanken et al. reported associations between salivary interleukin-1-beta and MS fatigue. ${ }^{61}$ It has generally been challenging to identify clinically useful cytokine biomarkers of inflammation in MS because cytokines are locally acting and short lived.

We examined CCL5 (also known as RANTES) and CCL18, which are cytokines from the chemokine family, and two adhesion molecules ICAM- 1 and VCAM- 1 to assess the role of inflammation. CCL5 is considered to be an inflammatory chemokine, whereas CCL18 is antiinflammatory; both have been implicated in MS lesions ${ }^{62-64}$; ICAM-1 and VCAM-1 are induced on immune cells and vascular endothelial cells of the blood-brain barrier by 
proinflammatory cytokines such tumor necrosis factor-alpha and interferon-gamma and facilitate the extravasation of inflammatory cells in MS; ICAM- 1 has been implicated in MS progression. ${ }^{65}$ We did not find any associations of these cytokines and inflammatory biomarkers. To further address the role of inflammation, we conducted linear regression analyses of FSS with T2-lesion volume (T2-LV) and gadolinium contrast-enhancing lesion number (CE-LN), which are widely used MRI measures of inflammatory disease burden and blood-brain breakdown in MS with age, sex, and the individual blood biomarker of interest as predictors. Neither T2-LV nor CE-LN was associated with the FSS dependent variable in these models (data not shown). The associations of HDL-C and TC to HDL-C ratio with FSS $\left(r_{p}=-0.36\right.$, $P=.006$ for HDL-C, and $r_{p}=.29, P=.032$ for TC to HDL-C ratio) and SFS $[\operatorname{Exp}(B)=0.95$ and $P=.015$ for HDL-C, and $\operatorname{Exp}(B)=2.68$ and $P=.025$ for TC to HDL$\mathrm{C}$ ratio] remained significant on adjustments for $\mathrm{T} 2-\mathrm{LV}$ and CE-LN (data not shown).

Additional limitations of this study include its crosssectional study design. A longitudinal study design would enable assessment of whether changes in HDL-C to TC ratio precede the fatigue changes. We also did not have an independent validation cohort, which would further strengthen the study findings.

In conclusion, our results indicate that cholesterol pathway biomarkers are associated with MS fatigue. Our research findings highlight a contribution of metabolic pathways in fatigue and raise the intriguing prospect that it may be possible to ameliorate MS fatigue via metabolic pathways independently of the MS disease process.

\section{Acknowledgments}

This is unfunded research. Support from the National Institute of Neurological Disorders and Stroke, United States (1R21NS098169) to the Ramanathan Laboratory is gratefully acknowledged.

Authors' contributions: R.W.B. contributed to data acquisition and interpretation, manuscript preparation, and critical revision of the manuscript for content. D.J. contributed to data acquisition and interpretation and critical revision of the manuscript for content. J.K. contributed to data acquisition and interpretation and critical revision of the manuscript for content. M.R. contributed to study concept and design, analysis and interpretation, critical revision of the manuscript for content, and study supervision. B.W.-G. contributed to critical revision of the manuscript for content. R.Z. contributed to analysis and interpretation and critical revision of the manuscript for content.

\section{Disclosures}

Richard Browne received research support from the National Institute of Health. Dejan Jakimovski has nothing to disclose. Jens Kuhle received speaker fees, research support, travel support, and/or served on advisory boards by ECTRIMS, Swiss MS Society, Swiss National Research Foundation, (320030_160221), University of Basel, Bayer, Biogen, Genzyme, Merck, Novartis, Protagen AG, Roche, and Teva Pharmaceuticals. Murali Ramanathan received research funding from the National Multiple Sclerosis Society, the Department of Defense, the National Institutes of Health and National Science Foundation, and Otsuka Pharmaceutical Development. Bianca Weinstock-Guttman received honoraria as a speaker and as a consultant for Biogen Idec, Teva Pharmaceuticals, EMD Serono, GenzymeSanofi, Novartis, and Acorda. Dr Weinstock-Guttman received research funds from Biogen Idec, Teva Pharmaceuticals, EMD Serono, Genzyme-Sanofi, Novartis, and Acorda. Robert Zivadinov received personal compensation from EMD Serono, Genzyme-Sanofi, Celgene, and Novartis for speaking and consultant fees. He received financial support for research activities from Genzyme-Sanofi, Novartis, Celgene, Mapi Pharma, and Protembis.

\section{References}

1. Fisk J, Pontefract A, Ritvo P, Archibald C, Murray T. The impact of fatigue on patients with multiple sclerosis. Can J Neurol Sci. 1994; 21:9-14.

2. Lerdal A, Celius E, Krupp L, Dahl A. A prospective study of patterns of fatigue in multiple sclerosis. Eur J Neurol. 2007;14:1338-1343.

3. Hadjimichael O, Vollmer T, Oleen-Burkey M. Fatigue characteristics in multiple sclerosis: the North American Research Committee on Multiple Sclerosis (NARCOMS) survey. Health Qual Life Outcomes. 2008;6:100.

4. Janardhan V, Bakshi R. Quality of life in patients with multiple sclerosis: the impact of fatigue and depression. J Neurol Sci. 2002;205: $51-58$.

5. Multiple Sclerosis Council for Clinical Practice Guidelines. Fatigue and Multiple Sclerosis: Evidence-Based Management Strategies for Fatigue in Multiple Sclerosis. Washington, DC: Paralyzed Veterans of America; 1998.

6. National MS Society and Paralyzed Veterans of America (PVA). Consumer Guide to Clinical Practice Guidelines Fatigue: What You Should Know, A Guide for People With Multiple Sclerosis. Minor updating in 2006 and 2015 by the National MS Society. 2019 National MS Society. Available at: https://www.nationalmssociety.org/NationalMSSociety/ media/MSNationalFiles/Brochures/Brochure-Fatigue-What-You-Sho uld-Know.pdf.

7. Krupp L, Alvarez L, LaRocca N, Scheinberg L. Fatigue in multiple sclerosis. Arch Neurol. 1988;45:435-437.

8. Braley TJ, Chervin RD. Fatigue in multiple sclerosis: mechanisms, evaluation, and treatment. Sleep. 2010;33:1061-1067.

9. Bakshi R, Shaikh ZA, Miletich RS, et al. Fatigue in multiple sclerosis and its relationship to depression and neurologic disability. Mult Scler. 2000;6:181-185.

10. Flachenecker P, Kumpfel T, Kallmann B, et al. Fatigue in multiple sclerosis: a comparison of different rating scales and correlation to clinical parameters. Mult Scler. 2002;8:523-526.

11. Motl RW, McAuley E, Wynn D, Suh Y, Weikert M. Effects of change in fatigue and depression on physical activity over time in relapsingremitting multiple sclerosis. Psychol Health Med. 2011;16:1-11.

12. Giovannoni G, Thompson AJ, Miller DH, Thompson EJ. Fatigue is not associated with raised inflammatory markers in multiple sclerosis. Neurology. 2001;57:676-681.

13. Cavallari M, Palotai M, Glanz B, et al. Fatigue predicts disease worsening in relapsing-remitting multiple sclerosis. Mult Scler. 2016;22: 1841-1849. 
14. Vaughn CB, Kavak KS, Dwyer MG, et al. Fatigue at enrollment predicts EDSS worsening in the New York State Multiple Sclerosis Consortium. Mult Scler. 2018;31352458518816619.

15. Chaudhuri A, Behan PO. Fatigue in neurological disorders. Lancet. 2004;363:978-988.

16. Rammohan KW, Rosenberg JH, Lynn DJ, Blumenfeld AM, Pollak CP, Nagaraja HN. Efficacy and safety of modafinil (Provigil) for the treatment of fatigue in multiple sclerosis: a two centre phase 2 study. $J$ Neurol Neurosurg Psychiatry. 2002;72:179-183.

17. Krupp L, Coyle P, Doscher C, et al. Fatigue therapy in multiple sclerosis: results of a double-blind, randomized, parallel trial of amantadine, permoline, and placebo. Neurology. 1995;45:1956-1961.

18. Asano M, Finlayson ML. Meta-analysis of three different types of fatigue management interventions for people with multiple sclerosis: exercise, education, and medication. Mult Scler Int. 2014;2014:798285.

19. Khalil M, Enzinger C, Langkammer C, et al. CSF neurofilament and $\mathrm{N}$-acetylaspartate related brain changes in clinically isolated syndrome. Mult Scler. 2013;19:436-442.

20. Barro C, Benkert P, Disanto G, et al. Serum neurofilament as a predictor of disease worsening and brain and spinal cord atrophy in multiple sclerosis. Brain. 2018;141:2382-2391.

21. Procaccini C, Santopaolo M, Faicchia D, et al. Role of metabolism in neurodegenerative disorders. Metabolism. 2016;65:1376-1390.

22. La Rocca C, Carbone F, De Rosa V, et al. Immunometabolic profiling of $\mathrm{T}$ cells from patients with relapsing-remitting multiple sclerosis reveals an impairment in glycolysis and mitochondrial respiration. Metabolism. 2017;77:39-46.

23. Browne RW, Weinstock-Guttman B, Horakova D, et al. Apolipoproteins are associated with new MRI lesions and deep grey matter atrophy in clinically isolated syndromes. J Neurol Neurosurg Psychiatry, 2014;85:859-864.

24. Weinstock-Guttman B, Zivadinov R, Horakova D, et al. Lipid profiles are associated with lesion formation over 24 months in interferon-beta treated patients following the first demyelinating event. J Neurol Neurosurg Psychiatry. 2013;84:1186-1191.

25. Weinstock-Guttman B, Zivadinov R, Mahfooz N, et al. Serum lipid profiles are associated with disability and MRI outcomes in multiple sclerosis. J Neuroinflammation. 2011;8:127.

26. Jakimovski D, Weinstock-Guttman B, Hagemeier J, et al. Walking disability measures in multiple sclerosis patients: Correlations with MRI-derived global and microstructural damage. J Neurol Sci. 2018; 393:128-134.

27. Krupp LB, LaRocca NG, Muir-Nash J, Steinberg AD. The fatigue severity scale. Application to patients with multiple sclerosis and systemic lupus erythematosus. Arch Neurol. 1989;46:1121-1123.

28. Kurtzke JF. Rating neurologic impairment in multiple sclerosis: an expanded disability status scale (EDSS). Neurology. 1983;33:1444-1452.

29. Beck AT, Steer RA, Brown GK. BDI ${ }^{\circledR}$ - FastScreen for Medical Patients. San Antonio, TX: Pearson; 2000.

30. Benedict RH, Fishman I, McClellan MM, Bakshi R, Weinstock-Guttman B. Validity of the beck depression inventory-fast screen in multiple sclerosis. Mult Scler. 2003;9:393-396.

31. Friedewald WT, Levy RI, Fredrickson DS. Estimation of the concentration of low-density lipoprotein cholesterol in plasma, without use of the preparative ultracentrifuge. Clin Chem. 1972;18:499-502.

32. Disanto G, Barro C, Benkert P, et al. Serum neurofilament light: A biomarker of neuronal damage in multiple sclerosis. Ann Neurol. 2017:81:857-870

33. Learmonth YC, Dlugonski D, Pilutti LA, Sandroff BM, Klaren R, Motl RW. Psychometric properties of the fatigue severity scale and the modified fatigue impact scale. J Neurol Sci. 2013;331:102-107.

34. Neitzer A, Sun S, Doss S, Moran J, Schiller B. Beck depression inventoryfast screen (BDI-FS): an efficient tool for depression screening in patients with end-stage renal disease. Hemodial Int. 2012;16:207-213.

35. Genest J, Frohlich J, Fodor G, McPherson R, Working Group on Hypercholesterolemia and Other Dyslipidemias. Recommendations for the management of dyslipidemia and the prevention of cardiovascular disease: summary of the 2003 update. CMAJ. 2003;169:921-924.
36. Millan J, Pinto X, Munoz A, et al. Lipoprotein ratios: physiological significance and clinical usefulness in cardiovascular prevention. Vasc Health Risk Manag. 2009;5:757-765.

37. Fellows K, Uher T, Browne RW, et al. Protective associations of HDL with blood-brain barrier injury in multiple sclerosis patients. $J$ Lipid Res. 2015;56:2010-2018.

38. Morra VB, Coppola G, Orefice G, et al. Interferon-beta treatment decreases cholesterol plasma levels in multiple sclerosis patients. Neurology. 2004;62:829-830.

39. Kos D, Kerckhofs E, Nagels G, D'Hooghe MB, Ilsbroukx S. Origin of fatigue in multiple sclerosis: review of the literature. Neurorehabil Neural Repair. 2008;22:91-100.

40. Mukhopadhyay S, Fellows K, Browne RW, et al. Interdependence of oxysterols with cholesterol profiles in multiple sclerosis. Mult Scler. 2017;23:792-801.

41. Gloire G, Legrand-Poels S, Piette J. NF-kappaB activation by reactive oxygen species: fifteen years later. Biochem Pharmacol. 2006;72: $1493-1505$.

42. Morgan MJ, Liu ZG. Crosstalk of reactive oxygen species and NFkappaB signaling. Cell Res. 2011;21:103-115.

43. Reid MB, Li YP. Tumor necrosis factor-alpha and muscle wasting: a cellular perspective. Respir Res. 2001;2:269-272.

44. Qiu J, Fang Q, Xu T, et al. Mechanistic role of reactive oxygen species and therapeutic potential of antioxidants in denervation- or fastinginduced skeletal muscle atrophy. Front Physiol. 2018;9:215.

45. Uchida T, Sakashita Y, Kitahata K, et al. Reactive oxygen species upregulate expression of muscle atrophy-associated ubiquitin ligase $\mathrm{Cbl}-$ b in rat L6 skeletal muscle cells. Am J Physiol Cell Physiol. 2018;314: C721-C731.

46. Balaban RS, Nemoto S, Finkel T. Mitochondria, oxidants, and aging. Cell. 2005;120:483-495.

47. Brites F, Martin M, Guillas I, Kontush A. Antioxidative activity of high-density lipoprotein (HDL): Mechanistic insights into potential clinical benefit. BBA Clin. 2017;8:66-77.

48. Navab M, Yu R, Gharavi N, et al. High-density lipoprotein: antioxidant and anti-inflammatory properties. Curr Atheroscler Rep. 2007; 9:244-248.

49. Barrientos G, Sanchez-Aguilera P, Jaimovich E, Hidalgo C, Llanos P. Membrane cholesterol in skeletal muscle: a novel player in excitationcontraction coupling and insulin resistance. J Diabetes Res. 2017; 2017:3941898.

50. Siebel AL, Heywood SE, Kingwell BA. HDL and glucose metabolism: current evidence and therapeutic potential. Front Pharmacol. 2015;6:258

51. Domingo-Espin J, Lindahl M, Nilsson-Wolanin O, Cushman SW, Stenkula KG, Lagerstedt JO. Dual actions of apolipoprotein A-I on glucose-stimulated insulin secretion and insulin-independent peripheral tissue glucose uptake lead to increased heart and skeletal muscle glucose disposal. Diabetes. 2016;65:1838-1848.

52. Lehti M, Donelan E, Abplanalp W, et al. High-density lipoprotein maintains skeletal muscle function by modulating cellular respiration in mice. Circulation. 2013;128:2364-2371.

53. Arai Y, Hirose N. Aging and HDL metabolism in elderly people more than 100 years old. J Atheroscler Thromb. 2004;11:246-252.

54. Landi F, Russo A, Cesari M, Pahor M, Bernabei R, Onder G. HDLcholesterol and physical performance: results from the ageing and longevity study in the Sirente geographic area (ilSIRENTE Study). Age Ageing. 2007;36:514-520.

55. Siller N, Kuhle J, Muthuraman M, et al. Serum neurofilament light chain is a biomarker of acute and chronic neuronal damage in early multiple sclerosis. Mult Scler. 2019;25:678-686.

56. Mainero C, Faroni J, Gasperini C, et al. Fatigue and magnetic resonance imaging activity in multiple sclerosis. J Neurol. 1999;246: 454-458.

57. Biberacher V, Schmidt P, Selter RC, et al. Fatigue in multiple sclerosis: associations with clinical, MRI and CSF parameters. Mult Scler. 2018;24:1115-1125. 
58. Marrie RA, Fisher E, Miller DM, Lee JC, Rudick RA. Association of fatigue and brain atrophy in multiple sclerosis. J Neurol Sci. 2005;228: 161-166.

59. Filippi M, Rocca MA, Colombo B, et al. Functional magnetic resonance imaging correlates of fatigue in multiple sclerosis. Neuroimage. 2002;15:559-567.

60. Fuchs TA, Vaughn CB, Benedict RHB, et al. Lower self-report fatigue in multiple sclerosis is associated with localized white matter tract disruption between amygdala, temporal pole, insula, and other connected structures. Mult Scler Relat Disord. 2018; 27:298-304.

61. Hanken K, Sander C, Qaiser L, et al. Salivary IL-1beta as an objective measure for fatigue in multiple sclerosis? Front Neurol. 2018;9:574.
62. Boven LA, Montagne L, Nottet HS, De Groot CJ. Macrophage inflammatory protein-1alpha (MIP-1alpha), MIP-1beta, and RANTES mRNA semiquantification and protein expression in active demyelinating multiple sclerosis (MS) lesions. Clin Exp Immunol. 2000;122:257-263.

63. Boven LA, Van Meurs M, Van Zwam M, et al. Myelin-laden macrophages are anti-inflammatory, consistent with foam cells in multiple sclerosis. Brain. 2006;129:517-526.

64. Takeshita Y, Ransohoff RM. Inflammatory cell trafficking across the blood-brain barrier: chemokine regulation and in vitro models. Іттиnol Rev. 2012:248:228-239.

65. Giovannoni G, Miller DH, Losseff NA, et al. Serum inflammatory markers and clinical/MRI markers of disease progression in multiple sclerosis. J Neurol. 2001;248:487-495. 


\section{Appendix}

Supplementary Table 1 Regression results for the FSS and SFS with sNfL, lipid profile and apolipoprotein profile biomarkers

\begin{tabular}{|c|c|c|}
\hline Biomarker & $\begin{array}{c}\text { FSS } \\
\text { Linear Regression } \\
r_{p}(P \text {-value })\end{array}$ & $\begin{array}{c}\text { SFS } \\
\text { Logistic Regression } \\
\operatorname{Exp}(B)(P \text {-value })\end{array}$ \\
\hline $\log _{10}$ transformed sNfL levels & $0.13(.31)$ & $2.16(.51)$ \\
\hline Total cholesterol (TC), mg/dL & $-0.078(.54)$ & $0.996(0.67)$ \\
\hline High density lipoprotein cholesterol (HDL-C), mg/dL & $-0.34(.006)$ & $0.95(.030)$ \\
\hline Low density lipoprotein cholesterol (LDL-C), mg/dL & $-0.063(.63)$ & $0.996(.67)$ \\
\hline Apolipoprotein A-I (ApoA-I), mg/dL & $-0.24(.058)$ & $0.98(.099)$ \\
\hline Apolipoprotein A-II (ApoA-II), mg/dL & $-0.19(.12)$ & $0.96(.31)$ \\
\hline Apolipoprotein B (ApoB), mg/dL & $0.16(.22)$ & $1.02(.25)$ \\
\hline Apolipoprotein C-II (ApoC-II), mg/dL & $0.17(.17)$ & $1.29(.17)$ \\
\hline Apolipoprotein E (ApoE), mg/dL & $0.14(.26)$ & $1.19(.36)$ \\
\hline Total cholesterol to $\mathrm{HDL}-\mathrm{C}$ ratio & $0.36(.004)$ & $3.55(.011)$ \\
\hline
\end{tabular}

All regression analyses adjusted for age, sex, EDSS and BDI-FS score, BMI, interferon-beta treatment status and statin treatment status.

The partial correlation $\left(r_{p}\right)$ values and the corresponding $P$-values are shown for each biomarker for FSS whereas $\operatorname{Exp}(B)$ and $P$-values are shown for the SFS.

Supplementary Table 2 Regression results for the FSS and SFS with cytokine and inflammatory biomarkers. All regression analyses adjusted for age, sex, EDSS and BDI-FS score

\begin{tabular}{|c|c|c|}
\hline Biomarker & $\begin{array}{c}\text { FSS } \\
\text { Linear regression } \\
r_{p}(P \text {-value })\end{array}$ & $\begin{array}{c}\text { SFS } \\
\text { Logistic regression } \\
\operatorname{Exp}(B)(P \text {-value })\end{array}$ \\
\hline $\log _{10}$ transformed CCL5 levels & $-0.12(.34)$ & $0.56(.42)$ \\
\hline $\log _{10}$ transformed CCL18 levels & $0.08(.52)$ & $3.02(.47)$ \\
\hline sICAM-1, $\mathrm{ng} / \mathrm{ml}$ & $0.18(.14)$ & $1.006(.38)$ \\
\hline sVCAM-1, ng/ml & $0.032(.80)$ & $1.000(.99)$ \\
\hline
\end{tabular}

CCL5: Chemokine (C-C motif) ligand 5; CCL18: Chemokine (C-C motif) ligand 18 (CCL18), sICAM-1: Soluble intercellular adhesion molecule-1; sVCAM-1: Soluble vascular adhesion molecule-1.

The partial correlation $\left(r_{p}\right)$ values and the corresponding $P$-values are shown for each biomarker for FSS whereas $\operatorname{Exp}(B)$ and $P$-values are shown for the SFS. 